\title{
DISPONIBILIDAD DE PIEZAS DE RECAMBIO EN LOS SERVICIOS POSTVENTA: UNA RECIENTE DISTORSIÓN LEGISLATIVA INTERNA CON EL DERECHO EUROPEO
}

\author{
Javier Avilés García \\ Catedrático de Derecho Civil \\ Universidad de Oviedo
}

\begin{abstract}
Resumen: Tras la entrada en vigor este 1 de marzo de 2021 de una serie de reglamentos de ejecución comunitarios que traen causa de la Directiva 2009/125/CE, 21 octubre (diseño productos), la configuración jurídica del nuevo "derecho a la reparación" de los bienes de consumo susceptibles de reparación ha dado un nuevo paso: la ampliación del plazo obligatorio de disponibilidad de las piezas de recambio en el mercado. Esta obligación temporal específica por cuenta de fabricantes, importadores o representantes autorizados se ha trasladado a nuestro país de una manera equívoca e inarmónica con la normativa europea, según la redacción dada al nuevo artículo 127 bis TRLGDCU. La reforma operada no distingue ni separa con nitidez lo que supone la reparación de los bienes de consumo como medida correctora específica por causa de la disconformidad con el contrato de compraventa -recogida en las Directivas 2019/770/UE y 2019/771/UE transpuestas-, y la reparación de los bienes de consumo entendida como medida general de política legislativa fundada en el ciclo de vida útil de esos bienes, incardinada ésta dentro del Plan de Acción europeo para la Economía Circular (2015, 2020), y que no estaba bajo el presupuesto habilitante en la transposición de ambas directivas.
\end{abstract}

Palabras clave: Derecho a la reparación de los bienes de consumo; servicios postventa; piezas de recambio; responsabilidad de fabricantes, importadores y distribuidores; durabilidad; índice de reparabilidad de los bienes; economía circular.

Title: Availability of spare parts in after-sales services: a recent internal legislative distortion with European law

Abstract: After the entry into force on March 1, 2021, of a series of community enforcement regulations that lead to Directive 2009/125/EC, October 21 (product design), the legal configuration of the new "right to repair" of consumer goods that can be repaired has taken a new step: the extension of the mandatory period of availability of spare parts on the market. This specific temporary obligation on behalf 
of manufacturers, importers or authorized representatives has been transferred to us in an equivocal and inharmonious way with European regulations, according to the wording given to the new article 127 bis TRLGDCU. The reform carried out does not clearly distinguish or separate what the repair of consumer goods entails as a specific corrective measure due to non-compliance with the contract of sale - included in the transposed Directives 2019/770/EU and 2019/771/EU (20 may)-, and the repair of consumer goods understood as a general measure of legislative policy based on the useful life cycle of those goods, included in the New Circular Economy Action Plan $(2015,2020)$, and which was not under the enabling budget for the transposition of both directives.

Keywords: Right to repair consumer goods; services post-sale; interchange parts; manufacturers, importers and distributors responsibility; durability; property repairability index; circular economy.

\section{SUMARIO. 1. DERECHO A LA REPARACIÓN DE BIENES DE CONSUMO Y PIEZAS DE RECAMBIO DISPONIBLES. 2. UNA DISTORSIÓN LEGISLATIVA INARMÓNICA CON EL DERECHO EUROPEO. 3. CONCLUSIONES}

En los últimos meses hemos podido leer en muchos medios de comunicación la noticia de la entrada en vigor en el ámbito de la UE de un nuevo "derecho a la reparación" de los bienes o productos de consumo, presentándola como una consecuencia de "una ley aprobada por el Parlamento Europeo en noviembre de 2020, que obliga a los fabricantes de neveras, lavadoras, secadores de pelo, televisores y otros productos de electrónica de consumo a garantizar que sus electrodomésticos puedan repararse durante al menos un periodo de diez años" ${ }^{1}$.

Asimismo, se ha publicitado que desde este pasado 1 de marzo de 2021 este derecho a reparar "obliga a las empresas (fabricantes, importadores y distribuidores 0 representantes autorizados) a que sus electrodomésticos puedan ser reparados durante al menos 10 años desde la fecha de su compra"2. En fin, se ha invocado en los medios muy equívocamente y de manera generalizada del amparo de una "ley" para referirse realmente a una "resolución" del Parlamento Europeo de 25 de noviembre de 2020 ("Hacia un mercado único más sostenible para las empresas y los consumidores"), y se ha hablado de un nuevo plazo obligatorio mínimo de disponibilidad para las piezas de recambio en los servicios postventa de 10 años, cuestiones ambas que como pasamos a ver no se corresponden exactamente con la realidad.

\footnotetext{
1 Por todos véase, por ejemplo, en La Vanguardia, Así te afecta el nuevo derecho europeo a reparar electrodomésticos, 8 marzo 2021, <https://www.lavanguardia.com/vivo/como-hacerdiy/20210305/6263735/derecho-reparar-ue.html>. Esta idea ha aparecido difundida en conexión directa con la sostenibilidad, como medio específico de lucha contra la obsolescencia técnica o programada de los bienes, con el objetivo de facilitar la reparabilidad de estos mediante un consumo más responsable que rompa con la práctica tan extendida de usar y tirar, para lo que es imprescindible disponer de las piezas de recambio a precios razonables.

2 Por todos véase, por ejemplo, Libre Mercado, La UE aprueba el "derecho a reparar" para que los electrodomésticos duren 10 años, 21 marzo 2021, <https://www.libremercado.com/2021-03-09/ueobligara-fabricantes-crear-productos-puedan-ser-desmontables-reparables-6716930/> .
} 
En efecto, vemos como en nuestro país el Real Decreto-ley 7/2021, de 28 de abril (Título VIII), con el objetivo de transponer las directivas de contratos de compraventa de bienes y de suministro de contenidos o servicios digitales (Directivas 2019/770/UE y 2019/771/UE, de 20 mayo), ha aprovechado también la ocasión para introducir en el TRLGDCU una reforma en los servicios postventa en lo que afecta al importante plazo de disponibilidad de piezas de recambio de los bienes de consumo por cuenta de fabricantes, importadores o representantes autorizados, lo que pudiera parecer algo de escasa trascendencia práctica, pero que realmente no es así. Pues bien, esta reforma se ha limitado sustantivamente a multiplicar por dos el plazo mínimo establecido en España para exigir esa disponibilidad de piezas de recambio (a contar desde la fecha en que el bien o modelo deje de fabricarse), que hasta ahora era de 5 años, para fijarlo en un nuevo plazo general mínimo de 10 años, sin que el Gobierno haya estimado conveniente realizar distinción alguna al respecto, esto es, sin atender a la diferente naturaleza o categoría de los bienes o aparatos sobre los que recae esta obligación decenal mínima por cuenta de fabricantes, importadores o distribuidores o representantes autorizados (art. 127 bis TRLGDCU).

\section{Derecho a la reparación de bienes de consumo y piezas de recambio disponibles}

Este cambio legislativo, según el propio Real Decreto-ley $7 / 2021$ pretende justificarse en la implementación parcial de un nuevo "derecho a la reparación" en lo tocante a la ampliación del plazo de disponibilidad sobre las piezas de repuesto (durabilidad), pues en su exposición de motivos se arguye como presupuesto habilitante de esta nueva regulación temporal invocando tanto la existencia de una resolución del Parlamento Europeo de 25 de noviembre de $2020^{3}$, como un informe sobre "Estrategia Española de Economía Circular 2030" en línea con esta 4 . Sin embargo, a nuestro juicio, desde una correcta técnica legislativa, debe entenderse más bien que esta reforma del nuevo plazo mínimo obligatorio de disponibilidad de piezas de repuesto trae causa directa, no del contenido de ambas directivas objeto de transposición ni tampoco de la resolución antedicha del Parlamento Europeo de 25 noviembre de 2020 o en ese informe estratégico, sino que más bien la ampliación de este nuevo plazo mínimo de hasta 10 años supone una mera actualización parcial de nuestra normativa estatal en materia de "reparación en los servicios postventa". Comprobamos, además, y esto es lo importante, que la razón sustantiva de este cambio legal del plazo mínimo de disponibilidad para las piezas de recambio de bienes de consumo, que está regulado en varios Reglamentos de ejecución de fecha de 1 de octubre de 2019, no aparece ni tan siquiera mencionada en la exposición de motivos del Real Decreto-ley 7/2021 que debiera tratar de justificarla (presupuesto

\footnotetext{
${ }^{3}$ Cfr. Exposición de Motivos Real Decreto-ley 7/2021, p. 25, que invoca esta resolución del Parlamento Europeo de 25 de noviembre de 2020 ("Hacia un mercado único más sostenible para las empresas y los consumidores", P9_TA (2020)0318), que no puede ser en modo alguno objeto de transposición. Su contenido trata de una serie de estrategias y medidas generales presentadas al Consejo y la Comisión para una próxima implementación normativa en el ámbito europeo.

4 Cfr. España circular 2030, Estrategia Española de Economía Circular (EEEC), en <https://www.miteco.gob.es/es/calidad-y-evaluacion-ambiental/temas/economia-

circular/espanacircular2030_def1_tcm30-509532.PDF>, documento inspirado en el "Nuevo Plan de Acción para la economía circular por una Europa más limpia y más competitiva", COM (2020)0098, 11 marzo 2020.
} 
habilitante del art. 86.1 CE), y que, finalmente, ha ampliado ese plazo de 5 años a los 10 años, haciéndolo además de manera general e indiscriminada para todo tipo de bienes o productos de consumo.

Pues bien, la clave de esta actualización normativa tiene su último fundamento en la aprobación de los cinco Reglamentos de ejecución de la UE, todos ellos de fecha de 1 de octubre de 2020, y que fueron publicados a la vez en el DOUE de 1 de octubre de 2020, y que comprobamos han pasado desapercibidos -no ya solo para muchos medios de comunicación especializados-, sino también para el mismo legislador interno (en este caso el Gobierno), que no ha llegado a justificar con claridad el contenido y alcance de esta ampliación generalizada a los 10 años como plazo obligatorio mínimo de disponibilidad sobre cualesquiera piezas de recambio por cuenta de fabricantes, importadores o distribuidores, y que entendemos no concuerda con la normativa europea en vigor desde el 1 de marzo de 2021.

Debe subrayarse que fueron precisamente estos cinco Reglamentos los que impusieron a fabricantes, importadores o representantes autorizados, para todo el ámbito de la UE, que, a partir del 1 de marzo de 2021, será obligatorio tener una disponibilidad efectiva de las piezas de recambio para toda una serie de bienes o aparatos de consumo de uso doméstico muy generalizados. Y la calificamos de "efectiva" por cuanto que el legislador europeo ha dejado regulados aspectos muy relevantes para su ejercicio tanto para profesionales como para consumidores, como son, por ejemplo, aquellos que afectan al medio de acceso a la información sobre reparación y mantenimiento de los bienes, los plazos máximos para realizar su entrega, o, también, la exigencia de mantener la disponibilidad de actualizaciones de software o firmware cuando se trate de bienes o productos de consumo con elementos digitales. Con todo, se observa que la nueva regulación en España dentro del nuevo artículo 127 bis TRLGDCU ("en todo caso" afirma) ha sido realizada con carácter general e indiscriminado para cualesquiera bienes o productos de consumo y por un plazo mínimo de 10 años, lo que se percibe como causa más que probable de un claro desajuste o inarmonía con la normativa europea de 2019, al margen de que se ha desaprovechado la ocasión para siquiera detallar, al menos, algunos de los nuevos contenidos recogidos genéricamente en la normativa europea de 2019 para el ejercicio efectivo de este nuevo derecho de disponer en plazo de las piezas de recambio. Veámoslo.

\section{Una distorsión legislativa inarmónica con el Derecho europeo}

En efecto, en el caso de España, el Real Decreto-ley 7/2021 (Título VIII) dispone ahora que esta obligación específica de disponibilidad de piezas de recambio para los bienes de consumo en los servicios postventa será "en todo caso" por un plazo mínimo de 10 años, constatándose que lo hace de manera general e indiscriminada para todo tipo de bienes o aparatos de consumo, sin diferenciar categorías o grupos de bienes (art. 127 bis, 1 TRLGDCU). De ahí que entendamos que con ello se produce una neta desarmonía con lo ya regulado por la normativa europea de 2019 con efectos de 1 de marzo de 2021. Así, yendo a los pormenores prácticos en esta cuestión clave de las piezas de repuesto, vemos que esto no concuerda con la normativa reglamentaria europea citada, donde se regula esta disponibilidad de las 
piezas de recambio (por el momento diseñada para bienes energéticos de uso doméstico) hasta en tres plazos distintos, según fuere la naturaleza o categoría de los bienes o aparatos sobre los que recaiga, con tres plazos distintos de disponibilidad mínima de 7, 8 y hasta 10 años, lo que no concuerda con los 10 años de nuestra norma de plazo único y exclusivo para todos los bienes de consumo ${ }^{5}$.

Comentamos que esta regulación "por el momento" está circunscrita a una serie de aparatos, pues es conocido que la propia Comisión ha puesto en marcha una ampliación a otra gama de bienes distintos a los contemplados en esos cinco reglamentos de 2019, todos relacionados con el consumo de energía. La normativa comunitaria en vigor ha regulado obligatoriamente esta obligación de disponibilidad de piezas de recambio por cuenta de fabricantes, importadores o representantes autorizados, por plazos distintos, aunque solamente lo hace para una amplia serie de bienes o productos relacionados con el consumo energético, pues todo ello se hace de conformidad con la Directiva 2009/125/CE de diseño ecológico, pero sin olvidar que lo hace para todo un amplio espectro de bienes de consumo, tal vez los más comunes de uso doméstico generalizado por el consumidor medio: aparatos de refrigeración, pantallas electrónicas, lavavajillas, lavadoras y lavadoras-secadoras eléctricas y, finalmente, añadiendo la especificación de aparatos de refrigeración con función de venta directa ${ }^{6}$.

En cuanto a la perdida de oportunidad en España para haberse detallado en el ámbito estatal (unidad de mercado), siquiera fuese de algunos de los nuevos contenidos de esta disponibilidad efectiva de piezas de recambio, téngase en cuenta que la nueva normativa europea de 2019 puntualiza mucho más allá de esos nuevos plazos mínimos obligatorios (7, 8 y 10 años), aportando una minuciosa serie de aspectos y circunstancias que podrían fácilmente haberse puntualizado dentro de un nuevo capítulo específico del TRLGDCU sobre los "servicios postventa y las piezas de recambio", a continuación del capítulo actual dedicado a la regulación de la "garantía legal y comercial de los bienes de consumo".

Profundizando un poco en esta ocasión perdida, esquemáticamente observamos que hay dos grandes bloques de contenidos, que hubieran podido contribuir a aclarar esta ampliada obligación temporal mínima para piezas de recambio por 10 años de

\footnotetext{
5 Cfr. nota 6, que recoge los plazos de cada tipo o clase de aparato.

${ }^{6}$ Cfr. Reglamento (UE) 2019/2019, 1 octubre 2019, por el que se establecen los requisitos de diseño ecológico aplicables a los aparatos de refrigeración, de conformidad con la Directiva 2009/125/CE del Parlamento Europeo y del Consejo [7 o 10 años, según tipo de piezas]; Reglamento (UE) 2019/2021, 1 octubre 2019, por el que se establecen requisitos de diseño ecológico aplicables a las pantallas electrónicas, de conformidad con la Directiva 2009/125/CE del Parlamento Europeo y del Consejo [7 u 8 años]; Reglamento (UE) 2019/2022, 1 octubre 2019, por el que se establecen los requisitos de diseño ecológico aplicables a los lavavajillas domésticos de conformidad con la Directiva 2009/125/CE del Parlamento Europeo y del Consejo [7 o 10 años, según tipo de piezas]; Reglamento (UE) 2019/2023 de la Comisión, 1 octubre 2019, por el que se establecen requisitos de diseño ecológico aplicables a las lavadoras domésticas y a las lavadoras-secadoras domésticas, de conformidad con la Directiva 2009/125/CE del Parlamento Europeo y del Consejo [10 años]; Reglamento (UE) 2019/2024 de la Comisión, 1 octubre 2019, por el que se establecen requisitos de diseño ecológico para los aparatos de refrigeración con función de venta directa de conformidad con la Directiva 2009/125/CE del Parlamento Europeo y del Consejo [8 años]. En sus respectivos artículos 2 se contienen las definiciones que determinan, caso por caso, qué tipo de bienes concretos son los que se incluyen dentro de cada tipo genérico de bien o producto (DOUE L 315, 5 diciembre 2019).
} 
responsabilidad para fabricantes, importadores y representantes autorizados: $1^{\circ}$ ) Compendio de requisitos sobre la utilización eficiente de los recursos, entre los que se incluyen: a) disponibilidad mínima de las piezas de recambio; b) acceso a la información sobre la reparación y el mantenimiento; c) plazo máximo para la entrega de las piezas de recambio solicitadas; d) requisitos de desmontaje para la valoración y el reciclaje de los materiales al tiempo que se evita la contaminación. Y $2^{\circ}$ ) compendio de requisitos sobre la información sobre reparación contenidas en los manuales de instrucciones para instaladores y usuarios finales y páginas web de libre acceso de los fabricantes, importadores o representantes autorizados. En este punto, en lo que afecta a las piezas de recambio se recoge, por ejemplo, la información sobre el acceso a una reparación profesional de un bien (sitios web, direcciones y datos de contacto); la información pertinente para el encargo de piezas de recambio directamente $o$ a través de otros canales facilitados por fabricantes, importadores 0 representantes autorizados; o el periodo mínimo en el que deben estar disponibles las piezas de recambio necesarias para la reparación de un aparato determinado.

Así todo, en cuanto al contenido de esta puesta a disposición de las piezas de recambio, dejamos al margen otras muchas especificaciones sobre piezas de recambio reguladas según el grupo o categoría de aparatos o productos de que se trate, como son, por ejemplo, la información sobre la fecha de introducción en el mercado de la primera unidad de un modelo o de un modelo equivalente; la información gratuita que debe estar siempre a disposición de terceros (incluidos operarios de mantenimiento, intermediarios y proveedores de piezas de recambio) que se dediquen a la reparación o reutilización profesional de tales aparatos; o el acceso gratuito o a un coste justo, transparente y no discriminatorio de la última versión disponible en el mercado de software y firmware, así como la disponibilidad garantizada de actualizaciones de software y firmware por un plazo mínimo de tiempo; etc. ${ }^{7}$

En suma, la diferenciación de plazos mínimos de la normativa comunitaria para las piezas de recambio ( 7,8 y 10 años), adaptada a cada categoría o tipo de bienes o aparatos relacionados con la energía según la Directiva de diseño ecológico de 2009 (Directiva 2009/125/CE), pudiera parecer infundada o de una importancia relativa, pero no es así, por cuanto que los estudios que lo han precedido, de conformidad con los parámetros fijados en esta directiva, han sido muy largos y rigurosos. Lo que sí parece llamativo es que el legislador nacional no haya tenido en cuenta en absoluto esos diversos bienes o tipos de aparatos y sus plazos, pues tales plazos de 7, 8 o 10 años, se han estudiado y luego establecido de conformidad con la Directiva de diseño de $2009^{8}$, con base en informes y estudios directamente "relacionados con la energía (y) que representan un volumen notable de ventas y de comercio en la Unión", tras el análisis previo que llevó a la conclusión de que "todos (los bienes o aparatos seleccionados) tienen un impacto medioambiental importante y que, por su diseño, revelan un gran potencial de mejora con relación a dicho impacto, sin que ello conlleve costes excesivos".

\footnotetext{
7 Cfr. Anexo II, Apartado E, Reglamento (UE) 2019/2021, 1 octubre 2019, por el que se establecen requisitos de diseño ecológico aplicables a las pantallas electrónicas, de conformidad con la Directiva 2009/125/CE del Parlamento Europeo y del Consejo.

${ }^{8}$ Cfr. Directiva 2009/125/CE.
} 
En efecto, nuestro artículo 127 bis TRLGDCU olvida y no tiene en cuenta que la nueva reglamentación comunitaria en esta materia, en vigor desde 1 de marzo de 2021, parte de un laborioso estudio previo de diseño ecológico de cada producto, donde se han seleccionado por separado aquellos bienes o aparatos que deben considerarse "prioritarios" para así adoptar individualizadamente aquellas medidas de ejecución más adecuadas "para cada grupo de ellos diferenciados". Todo esto explica mejor y justifica fácilmente la existencia de Reglamentos distintos e independientes, cada uno con sus particularidades y sus plazos distintos (7, 8 y 10 años), para lo que se han tenido en cuenta tanto "los aspectos técnicos, medioambientales y económicos" de cada tipo o categoría de bienes o aparatos, "así como el comportamiento de los usuarios en la práctica", lo que ha exigido - y esto no es baladí- que cada grupo o categoría de aparatos haya sido "objeto de un Reglamento independiente sobre diseño ecológico"9 10 .

Así todo, por un lado, comprobamos que los distintos plazos mínimos obligatorios para las piezas de recambio establecidos por los cinco reglamentos de ejecución, según la naturaleza de cada grupo o categoría de aparatos, son por el momento de 7, 8 o 10 años: aparatos de refrigeración, aparatos con pantallas electrónicas, lavavajillas, lavadoras y lavadoras-secadoras eléctricas, y aparatos de refrigeración con función de venta directa. Por otro lado, en nuestro país, el plazo general mínimo obligatorio, reiteramos, ha quedado fijado por el Real Decreto-ley 7/2021, de 28 de abril, con carácter uniforme para todas las piezas de recambio de cualquier tipo de bien de consumo, estén o no relacionados con la energía, en el plazo mínimo de los 10 años a contar desde la fecha en que el bien deje de fabricarse (art. 127 bis, 1 TRLGDCU, "en todo caso").

A nuestro juicio, y esto es a lo que queríamos llegar, se está distorsionando lo previsto por la normativa reglamentaria europea del año 2019 para todo el ámbito de la UE en esta materia, al no diferenciarse detalladamente los distintos plazos específicos ahí señalados en atención a las distintas categorías o grupos de bienes o productos de consumo de que se trate, a lo que no debe olvidarse se ha llegado tras una concienzuda reflexión con base en muy importante Directiva 2009/125/CE, incorporada literalmente al ordenamiento español mediante $R D 187 / 2011$, de 18 de febrero, relativo al establecimiento de requisitos de diseño ecológico aplicables a los productos relacionados con la energía.

Nos parece que esto podrá dar lugar a algunos serios problemas prácticos de abastecimiento en el suministro de muchas piezas de recambio por parte de muchas marcas de bienes o productos, con cargo a fabricantes, importadores o representantes autorizados en el seno de la Unión Europea. Téngase en cuenta la existencia en el mercado de modelos de productos que, por ejemplo, dejen de

\footnotetext{
${ }^{9}$ Véanse considerandos 1 a 5 y 10 de los cada uno de los Reglamentos de 1 de octubre de 2019, publicados en el DOUE L 315, de 5 de diciembre de 2019.

${ }^{10}$ Sin entrar en mayores detalles, es pertinente apuntar que es la propia Comisión quien ha previsto los pasos y plazos a seguir para la propia revisión futura de tales Reglamentos, lo que se hará "a la luz del progreso tecnológico y presentará al Foro consultivo los resultados de dicha evaluación, en su caso, junto con un proyecto de propuesta de revisión de 25 de diciembre de 2025" (art. 8, Reglamento (UE) 2019/2019, sobre aparatos de refrigeración).
} 
fabricarse en aquellos Estados miembro que se guíen en su legislación interna -que será lo habitual- por esos tres plazos distintos previstos en tales reglamentos de ejecución (7, 8 y 10 años), y que, en consecuencia, los plazos mínimos de disponibilidad de las piezas de recambio de esos modelos sean más cortos que los ahora fijados "en todo caso" en nuestra legislación estatal, cuyo plazo mínimo obligatorio ha quedado fijado en los 10 años para cualesquiera piezas de recambio, sea de aparatos relacionados con la energía o no.

Parece importante y deseable invocar una uniformidad y armonización normativa de plazos mínimos de disponibilidad en esta materia, al menos para todos aquellos bienes con piezas de recambio que gozan de una idéntica regulación comunitaria prevista a partir del 1 de marzo de 2021 (cinco reglamentos mencionados). Entre todos ellos, valga apuntar de modo anecdótico que tan solo es el Reglamento (UE) 2019/2023, de 1 octubre, sobre piezas de recambio de lavadoras domésticas y lavadoras-secadoras, el único que recoge el plazo único de disponibilidad obligatoria de todos sus recambios por el plazo mínimo de 10 años, es decir, el único que coincide con el plazo mínimo común ahora exigido en España, si bien para todo tipo o categoría de bienes de consumo reparables con piezas de recambio.

Por otro lado, por lo que se refiere a la mera entrega de piezas de recambio para todo aquel que las hubiere solicitado, la nueva reglamentación europea obliga a que tal disponibilidad se formalice en un plazo nunca superior a los quince días laborales a partir del momento en que se haya recibido tal solicitud o encargo. Nada de esto se comenta en la nueva regulación del artículo 127 bis TRLGDCU, lo que evidencia claramente, de nuevo, que esta reforma no ha tenido en cuenta en absoluto esta normativa europea.

Cuestión complementaria, que también afecta al "derecho de reparación", y no menos relevante que los dos anteriores, y que sin embargo aparece pormenorizada en los cinco Reglamentos de 1 octubre de 2019, es la concreción del acceso a cualquier información sobre la reparación y mantenimiento de tales bienes, cuestión relevante que condiciona el ejercicio real del "derecho de reparación" para esos cinco tipos o categorías de bienes regulados para todo el ámbito europeo. En nuestro caso, con el nuevo artículo 127 bis TRLGDCU podrá interpretarse, como condición obligatoria para el cumplimiento de fabricantes, importadores y distribuidores, que este plazo mínimo de disponibilidad de 10 años para todo tipo de piezas de recambio lo es para cualquier aparato o electrodoméstico de uso doméstico, incluidos todos aquellos aparatos distintos de esas cinco categorías de bienes relacionadas con la energía de los reglamentos comunitarios, extendiéndose asimismo a todos aquellos supuestos tan relevantes como son los que afectan a las piezas de recambio de los vehículos automóviles, que gozan en España de una regulación especial (RD 1457/1986, 10 enero), arcaica y necesitada de una reforma integral.

A mayor abundamiento, en materia de información precontractual, esta nueva regulación comunitaria, en vigor desde 1 de marzo de 2021, obliga a que los listados de piezas de recambio de los distintos aparatos o bienes de consumo (o sus componentes), así como el procedimiento para encargarlas o las correspondientes instrucciones de reparación e instalación, sean públicamente accesibles a través del 
sitio web de libre acceso de cada fabricante, importador o representante autorizado de cada bien o producto de consumo, lo que debe ser así desde el mismo momento de la introducción en el mercado de la primera unidad o modelo introducido hasta el fin de cada uno de distintos plazos de disponibilidad previstos.

En este sentido, como no podía ser de otra forma, se posibilita que el acceso efectivo por parte de los reparadores profesionales a esa información específica sobre reparación y mantenimiento de los aparatos, a lo largo de su vida útil, se realice a más tardar a partir de los dos años de la introducción en el mercado de la primera unidad de cada modelo específico de aparato de que se trate, y ello hasta el fin del plazo de disponibilidad fijado legalmente en cada listado de piezas de recambio $(7,8$ y 10 años $)^{11}$. Conviene advertir, una vez más, que esta regulación está vigente desde este pasado 1 de marzo de 2021 para todos los productos antedichos relacionados con la energía, de conformidad con los parámetros legales establecidos en la Directiva $2009 / 125 / C E$, y que son los contenidos y regulados en esos cinco reglamentos de ejecución antedichos, todos ellos de fecha de 1 de octubre de $2019^{12}$.

Por su relación con el acceso a las piezas de repuesto, merece particular atención considerar que en esta nueva regulación comunitaria sobre las piezas de recambio se deja ahora plena libertad al fabricante, importador o representante autorizado para establecer un proceso rápido para contestar a las solicitudes de incorporación a un registro de reparadores profesionales ( 5 días laborables), estableciéndose este mecanismo como un requisito indispensable para poder acceder a la información sobre la reparación y mantenimiento de los bienes de consumo. Comprobamos, no obstante, que este nuevo mecanismo queda sujeto a esta triple comprobación por parte de los fabricantes titulares del registro: a) comprobación sobre la competencia técnica de suficiencia profesional del reparador para la realización de las tareas específicas (basta con el cumplimiento de la reglamentación exigida a los profesionales en cada Estado miembro en que se opera; cabe sustituirlo por un sistema de registro oficial de reparador profesional en caso de que éste exista en un Estado miembro); b) comprobación sobre si el reparador profesional está protegido por un seguro profesional adecuado para el desempeño de cada actividad de reparación, al margen de que este sea o no exigible legalmente dentro de cada Estado miembro; y c) posibilidad de imponer, a cambio de la incorporación a este registro, unos cánones razonables, que en ningún caso desincentiven, para con ello poder acceder a la información que se necesite para la reparación o para recibir actualizaciones periódicas necesarias para el bien o producto que lo precise.

En último término, en continuidad con este nuevo "derecho a reparar", ligado estrechamente a la referida disponibilidad y acceso a las piezas de recambio, también con fecha de 1 de marzo de 2021, esos cinco reglamentos de 1 de octubre de 2019, redactados de conformidad con la Directiva 2009/125/CE (diseño productos),

\footnotetext{
${ }^{11}$ Con carácter especial, téngase en cuenta que, por ejemplo, para el caso de la categoría de pantallas electrónicas, es preciso, respecto a terceros que se dediquen a la reparación y reutilización de pantallas, tener la disponibilidad de actualizaciones del software y firmware, y esto de manera gratuita o a un coste justo, información transparente y no discriminatoria durante un periodo de 8 años a partir de la introducción en el mercado de la última unidad de un modelo de producto dado (Reglamento (UE) 2019/2021, 1 octubre 2019, por el que se establecen requisitos de diseño ecológico aplicables a las pantallas electrónicas, Anexo II, Requisitos de diseño ecológico, Apartado E).

12 Cfr. DOUE L 315, de 5 de diciembre de 2019.
} 
establecen como obligación informativa que es preciso facilitar al consumidor en todos los supuestos, aquella que especifique en cada caso cuál es el período mínimo durante el cual será posible conseguir las piezas de recambio necesarias para cada bien o producto. Esta información deberá asimismo aparecer consignada en el sitio web de libre acceso del fabricante, importador o representante autorizado.

\section{Conclusiones}

Al margen del pasmo que supone toparse ante un Real Decreto-ley ómnibus a pesar de los tiempos que corren ${ }^{13}$, entendemos que se ha perdido la ocasión para afrontar en materia de servicios postventa y piezas de recambio, ligada al nuevo concepto de durabilidad de los bienes de una economía circular, para hacerlo con un carácter general e integrador con toda la normativa vigente en España, de conformidad con la legislación comunitaria en vigor desde este 1 de marzo de 2021 y con los proyectos en curso de la Comisión Europea. Aquí se separan claramente dos tipos de requisitos fundamentales a tener en cuenta de este nuevo "derecho a la reparación", que al menos podrían haberse apuntado o mencionado en el nuevo artículo 127 bis TRLGDCU: a) los requisitos que forman parte de la eficiencia en los recursos (disponibilidad y acceso a las piezas de recambio de los bienes, distinguiendo tipos de bienes o aparatos distintos como hacen los reglamentos europeos), y, b) los requisitos de información específicos de cada categoría o tipo de aparatos o bienes de consumo (donde vemos que siempre figura el tiempo mínimo en que deben estar disponibles las piezas de recambio de los bienes recogidos en los distintos reglamentos).

En suma, entendemos que para el correcto encauzamiento legal de este nuevo "derecho a la reparación" de los bienes de consumo en nuestro país, a nuestro juicio, es absolutamente indispensable que el legislador nacional separe conceptualmente con claridad -como no ha hecho el Real Decreto-ley $7 / 2021-$, entre la reparación de este tipo de bienes entendida como una medida correctora por disconformidad con el contrato de compraventa -recogida en ambas directivas de 2019 transpuestas-, y la reparación de los bienes de consumo entendida como una medida general de política legislativa fundada en el ciclo de vida útil de esos bienes, e incardinada dentro del Plan de Acción europeo para la economía circular $(2015,2020)$, y que no quedaba bajo el presupuesto habilitante del Real Decreto-ley $7 / 2021$ para la transposición de las Directivas 2019/770/UE y 2019/771/UE, de 20 de mayo.

Este nuevo "derecho a la reparación" de bienes debe comportar, sustantivamente, la adopción de medidas legislativas específicas de lucha contra la obsolescencia prematura, técnica o programada, lo que previsiblemente ocasionará problemas de interpretación con la indeterminada generalización establecida -sin matices- por un plazo mínimo obligatorio de 10 años del derecho a disponer de las piezas de repuesto

\footnotetext{
13 Tampoco hay mucha lógica por tantas prisas en la transposición de la que trae causa esta cuestión (8 meses antes de que terminase el plazo dado por ambas directivas), cuando resulta que la fecha de entrada en vigor de ambas no es hasta el 1 de enero de 2022 (art. 24 Directiva 2019/770/UE y 2019/771/UE), máxime cuando no hemos olvidado lo ocurrido con la muy relevante Directiva 2014/17/UE, de 4 de febrero de 2014, sobre los contratos de crédito celebrados con los consumidores para bienes inmuebles de uso residencial, transpuesta en España por Ley 5/2019, de 15 de marzo, reguladora de los contratos de crédito inmobiliario, que España incorporó con más de tres años de retraso en el grupo de países de cola formado por Croacia, Chipre y Portugal.
} 
por cuenta de fabricantes, importadores o distribuidores a través de los servicios postventa.

En este sentido, entendemos que ha llegado el momento de afrontar, de una vez por todas, la regulación pormenorizada de toda esta materia de los "servicios postventa" ligada a las "piezas de recambio" y a los imperativos de la economía circular y de lucha contra la obsolescencia prematura, técnica o programada de los bienes o productos energéticos o electrónicos. Para ello parece imprescindible actualizar pormenorizadamente cuanto antes toda esta materia dentro de un título específico dentro del TRLGDCU sobre los "servicios postventa", separado del dedicado a las "garantías" legales y comerciales, pero en estrecha conexión con este como parece lógico.

A nuestro juicio, por ejemplo, en este proyecto debería incorporarse buena parte de la arcaica y dispersa normativa de nuestro país, ahora incluida en el Real Decreto 58/1988, de 29 de enero, sobre protección de los derechos del consumidor en el servicio de reparación de aparatos de uso doméstico (arts. 3 y 4 sustantivamente), y en el Real Decreto 1457/1986, de 10 de enero, que regula la actividad industrial y la prestación de servicios en los talleres de vehículos automóviles de sus equipos y componentes (art. 9 sustantivamente, sobre las piezas de recambio), lo cual daría pie para actualizar normativamente todo lo relativo a las garantías actualmente asociadas a los servicios específicos de reparaciones, cuestión generadora de muchos conflictos y problemas interpretativos desde la puesta en marcha de la garantía legal de conformidad en España en el año 2003 (art. 6 RD 58/1988 y art. 16 RD 1457/1986). De este modo se unificaría una legislación de consumo que actualmente goza de una enorme dispersión, máxime por estar en muchos casos recogida en muchas legislaciones autonómicas, que debe necesariamente acomodarse con toda la normativa europea citada y con los proyectos en curso de ampliación del derecho a reparar para todos los bienes susceptibles de reparación, unidos a la durabilidad o vida útil del bien, más allá de su relación o no con la energía.

Pues bien, para armonizarse todo esto deberían aprovecharse igualmente todos los plazos ahora recogidos en los cinco Reglamentos comunitarios de 2019, para lo que habría que acometer una distinción legal clara entre los distintos tipos o categorías de bienes o productos (tipificación), de conformidad con las indicaciones de la legislación de la UE, al objeto de justificar la existencia de plazos mínimos de disponibilidad de piezas distintos (7, 8 y 10 años), con especial referencia a las piezas de recambio electrónicas y eléctricas, así como del acceso a todas aquellas actualizaciones del software y firmware que sean precisas para la puesta al día y mantenimiento de los bienes o aparatos con elementos digitales. A nuestro juicio, esta tarea de tipificación entre las distintas categorías de bienes, productos o tipos de aparatos debió acometerse una vez que se ha optado por la supresión de la arcaica y desubicada definición de los "productos de naturaleza duradera" (art. 126 TRLGDCU derogado), que fue redactada en el texto originario de la LGDCU dentro de un particular "régimen de comprobación, reclamación, garantía y posibilidad de renuncia o de evolución que se establezca en los contratos" ya inexistente y posteriormente derogado (antiguo art. 11 LGDCU). 
La actual iniciativa del Ministerio de Consumo de regular el llamado "índice de reparabilidad de aparatos eléctricos y electrónicos", según el modelo francés vigente desde el 1 de enero de 2021, podría ser el punto de apoyo para afrontar con rigor esta reforma global integradora del derecho a reparar asociada a los servicios postventa en nuestro país, que incluya, entre otras, todas las cuestiones aquí apuntadas que aparecen estrechamente ligadas también a la Estrategia Española de Economía Circular (España Circular 2030) y de la Agenda $2030^{14}$. Es reseñable que entre los cinco parámetros que sirven para el cálculo numérico de este "índice de reparabilidad", que pretende introducirse en el etiquetado de los bienes de consumo, se incluye la duración prevista en que estará disponible la documentación técnica relativa al mantenimiento del bien, y la duración en que estarán asimismo disponibles en el mercado las piezas de recambio a las que quieran acceder tanto reparadores como consumidores. Todo esto es argumento más que suficiente para que esta materia se incluya asimismo dentro de un nuevo título del TRLGDCU dedicado exclusivamente a la regulación pormenorizada y nítida e los "servicios postventa y las piezas de recambio", tan estrechamente conectada con los imperativos y nuevas exigencias de la economía circular respecto a los bienes de consumo.

\footnotetext{
${ }^{14}$ Esta iniciativa del Ministerio de Consumo es una copia casi idéntica a la aprobada por el artículo 16 de la Ley francesa no 2020-205, de 10 de febrero 2020, sobre la lucha contra los residuos y la economía circular, completada por el Decreto no 2020-1757, sobre el índice de reparabilidad de los equipos eléctricos y electrónicos y su correspondiente reglamento de desarrollo contenido en la Orden relativa a métodos de visualización, señalización y parámetros generales para el cálculo del índice de reparabilidad, ambos publicados el 29 de diciembre de 2020, con entrada en vigor el 1 de enero de 2021.
} 